\title{
Empfehlungen der OEGGG, ÖGUM und ÖGPPM zur Einhaltung der Standards bei erweiterter Ultraschalldiagnostik
}

\author{
Martin Haeusler ${ }^{1}$, Wolfgang Arzt ${ }^{2}$, \\ Erich Hafner ${ }^{3}$, Horst Steiner ${ }^{4}$ \\ ${ }^{1}$ MedUni Graz \\ 2 OEGGG, AG Bildgebende Verfahren; Ös- \\ terr. Ges. für Prä- und Perinatale Medizin \\ ${ }^{3}$ ÖGUM AK Geburtshilfe / Gynäkologie \\ ${ }^{4}$ ÖGUM-Präsident
}

Die Forderung der österreichischen Fachgesellschaften weist schon lange auf die Bedeutung einer Differenzierung der ersten und 2. Ultraschalluntersuchung in eine Basisuntersuchung und eine erweiterte Untersuchung hin $(1,2)$. In die letzte Mutterkindpass-Version (2009) wurde diese wichtige Forderung aufgenommen. Dies ist sowohl für die Ärzte/innen als auch für die Schwangeren von Vorteil, weil dadurch klar wird, dass nicht jede/ $r$ alles können muss und besondere Untersuchungen ein besonderes Wissen erfordern. Es ist heute üblich und ganz normal geworden, Schwangere für eine spezielle Untersuchung an eine spezialisierte Stelle zu überweisen. Die Information der Schwangeren über diese Untersuchungsmöglichkeiten ist integraler Bestandteil der Schwangerenbetreuung und ermöglicht den Frauen die Entscheidung, ob sie eine erweiterte Untersuchung, die ja oft keine Kassenleistung ist, in Anspruch nehmen wollen.

\section{1) Ersttrimester-Screening, Combined Test

Über die Möglichkeit des ErsttrimesterScreenings sollte jede Schwangere, unabhängig von ihrem Alter, informiert werden (MKP, 1. Ultraschalluntersuchung, „Aufklärung über weitere pränataldiagnostische Untersuchungen erfolgt“). Zum Ersttrimester-Screening gehört eine anatomische Beurteilung des Feten, auf Wunsch auch eine Blutabnahme mit Bestimmung zweier Hormonparameter zur Wahrscheinlichkeitsberechnung auf Trisomien, der Überbegriff „Combined Test“ hat sich dafür etabliert. Es ist eine komplexe Untersuchung, die seit den ersten Publikationen (3-5) immer weiter verfeinert wurde (6).

Es geht dabei um die Berechnung des Trisomie-Risikos und damit um die Entscheidung für oder gegen eine invasive Abklärung. Daher ist eine ausreichende Qualität bei der Durchführung dieser Tests sehr wichtig: Unnötige Punktionen gefährden gesunde Feten, falsch negative Tests setzen die Untersucher/innen einem erheblichen Haftungsrisiko aus.

Um die Qualität der Combined Tests sicher zu stellen, wurden klare Qualitätskriterien von der FMF London und dann auch von der FMF Deutschland vorgegeben:

Der/die UntersucherIn muss qualifiziert sein, jeder muss selbst mit der entsprechenden Software die Berechnungen vornehmen, die Labors müssen zertifiziert sein.

Der/die UntersucherIn kann sich das Wissen mittlerweile über einen kostenfreien Internetkurs aneignen (http://www.fetalmedicine.com/fmf/), sich dann primär zertifizieren, die nötige Software gratis herunterladen und jährliche Audits absolvieren. Bei der FMF Deutschland ist der Prozess ähnlich (http://www.fmf-deutschland. info/de/arzt-info/), aber teils kostenpflichtig. Eine Auditierung/Zertifizierung/Akkreditierung erfolgt immer ad personam, das heißt, sie kann nicht einfach an einen Assistenten/in delegiert werden. Sie ist verpflichtend für alle, die die Leistung „Ersttrimesterscreening“ anbieten.

Es ist ganz klar, dass dies State of the Art und im Streitfall gutachterlich von großer Bedeutung ist.

Man trifft immer wieder auf folgende Ansichten, die den heutigen Standards völlig widersprechen und vor denen wir hiermit warnen möchten:

1. „Ich habe vor Jahren einen Kurs gemacht und mich zertifiziert, und das reicht ja wohl. Ich schicke die NT-Werte mit maternalem Blut an ein Labor, und die senden mir dann den kompletten Testbefund. Dadurch spare ich mir auch die Software und das lästige Herumrechnen".

2. „Ich habe vor Jahren einen Kurs gemacht und mich zertifiziert, und das reicht ja wohl. - Eine/ $r$ in meinem Spital hat die Auditierung und wir anderen arbeiten unter seinem/ihrem Namen“.

3. „Ich habe vor Jahren einen Kurs gemacht und mich zertifiziert, und das reicht ja wohl. - Ich mache Ersttrimesterschälle in meiner Praxis ohne Biochemie, und das geht bestens. Bin ja auch Facharzt/ärztin. Dafür kann ich durchaus einen angemessenen Preis verlangen“.

4. „Ich bin ein erfahrener Ultraschaller und brauche mich nicht überprüfen zu lassen. Ich messe exakt die NT und schätze die Wahrscheinlichkeit anhand einer Tabelle ein. Oder ich verwende einen fixen Grenzwert“.

Es muss klar gesagt werden, dass alle diese Varianten Verstöße gegen die Qualitätsstandards sind und die 3 Fachgesellschaften dringend davor warnen, so vorzugehen! 
Auch die Behauptung, die eingangs angeführten Qualitätsvorgaben seien in Österreich nicht verpflichtend, da von ausländischen Gesellschaften erstellt, wird im Klagsfalle kaum helfen. Es gibt in der Medizin Regeln, die sich aufgrund ihrer Logik und jahrelangen fachlichen Bewährung als internationaler Standard in Fachkreisen durchgesetzt haben. Hier braucht man mit der Umsetzung nicht darauf zu warten, bis die Ärztekammern oder das Ministerium dazu gesetzliche Regelungen erlassen. Im Gegenteil ist es als bahnbrechende Leistung anzuerkennen, dass hier fachinterne Qualitätsstandards erarbeitet wurden, ohne solange zu warten, bis Gerichtsurteile zum Handeln zwingen.

Es lässt sich einfach überprüfen, ob der/ die Untersucher/in aktuell zum Combined-Testen zugelassen ist: Die Liste der FMF London-Zertifizierten und Auditierten ist auf der Startseite der Homepage abrufbar: http://www.fetalmedicine. com/fmf/ („List of accredited doctors“ / nur die 2. Liste zeigt die Auditierten!) und bei der FMF Deutschland kann man unter http://www.fmf-deutschland.info/de/patienten-info/zertifizierte-gynaekologen/ die Ärzte namentlich suchen und dadurch deren Zulassung überprüfen.

Im Interesse der Schwangeren und in unserem eigenen Interesse erachten wir es also für wichtig, darauf hinzuweisen, dass CombinedTestsnurregelkonformzurAnwendung kommen dürfen. Über die Homepages der FMF London und Deutschland kann jede/ $\mathrm{r}$ kontrollieren, ob eine Zertifizierung und aktuelle Auditierung vorliegen.

\section{2) Organ-Screening (Detailultraschall)}

Auch für diese Untersuchung wurden Standards erarbeitet und publiziert (7-10), eine überarbeitete Version wurde im Dezember 2012 publiziert (11). Die erforderlichen Kenntnisse zum Organ-Screening entsprechen dem Stufe-II-Niveau der ÖGUM/DEGUM/SGUM. Es ist zwar nicht gefordert, dass man die Stufe-II-Qualifikation hat, um diese Untersuchung vorzunehmen, es müssen jedoch die Untersuchungsstandards eingehalten werden. Diese betreffen die Bildgebung, die Gerätequalität, die Bilddokumentation und Beratung. Natürlich ist damit auch ein entsprechender Zeitaufwand verbunden. Eine spezielle praktische und theoretische Aus- und Weiterbildung führt einen an diese Untersuchungsstandards he- ran. Im Schadensfall wird jeder dieser Punkte sachverständig zu beurteilen sein.

Das Organ-Screening anzubieten und abzurechnen, beinhaltet sowohl die Diagnostik erkennbarer Fehlbildungen als auch die Risikointerpretation von Softmarkern (12-16). Eine Schwangere also nach dem privat bezahlten „Organscreening“ wegen eines echogenen Fokus oder eines erweiterten fetalen Nierenbeckens einem Zentrum zur Risikoeinschätzung zuzuweisen und auf diesem Wege ein echtes Organ-Screening mit Gesamtübernahme des Haftungsrisikos zu erreichen, ist keine korrekte Vorgangsweise und muss abgelehnt werden. Erst wenn im Rahmen des privat bezahlten Organ-Screenings echte Auffälligkeiten diagnostiziert wurden, soll an ein übergeordnetes Ultraschallzentrum weiter verwiesen werden.

Zu warnen ist auch vor verbalen Phantasiegebilden wie „Organscreening light“ oder „Organscreening Stufe 1“, die in falscher Sicherheit wiegen. Sobald der Name „Organscreening“, „erweiterter Ultraschall“ oder „Detailultraschall“" verwendet wird, müssen die o.g. Qualitätskriterien nachweislich eingehalten werden.

\section{Literatur}

Ersttrimester-Screening

1 Erste österreichische Publikation mit Hinweis aufZertifizierungserfordernis: Arzt W; Bernaschek G; Brezinka C; Deutinger J; Hafner E; Häusler M; Krampl E; Schuchter K; Schwärzler P; Staudach A; Steiner H. Konsensuspapier: Empfohlene Screening-Untersuchungen für chromosomale und strukturelle Fehlbildungen. Speculum. 2002; 20: 6-9

2 Konsensusstatement zum Combined Test in Österreich: Häusler M, Husslein P, Leodolter S, Speiser P, Stummvoll W, Krampl E, Steiner H, Deutinger J, Auer B, Braune G, Hochmaier $\mathrm{G}$, Lassmann R. Ultraschall in der Schwangerschaft. Eine Information der OEGGG, der ÖGUM, der ÖGPPM und der ÖÄK. Speculum. 2007; 25:15-16

3 Erste Publikation über Nackentransparenz: Szabó J, Gellén J. Nuchal fluid accumulation in trisomy-21 detected by vaginosonography in first trimester. Lancet. 1990; 336 : 1133.

4 Erste Publikation von Prof. Nicolaides über Nackentransparenz: Nicolaides KH, Azar G, Byrne D, Mansur C, Marks K. Fetal nuchal translucency: ultrasound screening for chromosomal defects in first trimester of pregnancy. BMJ 1992; 304 : 867-869

5 Erste Publikation über den Combined Test: Spencer K, Souter V, Tul N, Snijders R, Nicolaides $\mathrm{KH}$. A screening program for trisomy 21 at 10-14 weeks using fetal nuchal translucency, maternal serum free beta-hu- man chorionicgonadotropin and pregnancyassociated plasma protein-A. Ultrasound Obstet Gynecol. 1999 Apr.;13: 231-237)

6 Combined Test und neue Entwicklungen (Combined Test und Pränatest): Benn P, Cuckle $\mathrm{H}$, Pergament E. Non-invasive prenatal diagnosis for Down syndrome: the paradigm will shift, but slowly. Ultrasound Obstet Gynecol; 2012; 39: 127-130. doi: 10.1002/uog.11083

\section{Organscreening-Standards}

7 Merz E. DEGUM-Standards in Prenatal Level II Ultrasound Diagnosis (18-22 weeks of Gestation). Ultraschall in Med. 2001; 22:199.

8 Merz E, Eichhorn KH, Hansmann M, Meinel K. Qualitätsanforderungen an die weiterführende differentialdiagnostische Ultraschalluntersuchung in der pränatalen Diagnostik (DEGUM Stufe II) im Zeitraum 18 bis 20 Schwangerschaftswochen. Ultraschall inMed. 2002; 23:11-12.

9 Arzt W; Krampl-Bettelheim E; Steiner H. Leitlinien der OEGGG, der ÖGUM und der ÖGPPM für die Durchführung von Ultraschalluntersuchungen in der Schwangerschaft. Speculum 2009; $27: 20-23$.

10 Salomon LJ, Alfirevic Z, Berghella V, Bilardo C, Hernandez-Andrade E, Johnsen SL, Kalache $\mathrm{K}$, Leung $\mathrm{KY}$, Malinger $\mathrm{G}$, Munoz $\mathrm{H}$, Prefumo F, Toi A, Lee W; ISUOG Clinical Standards Committee. Practice guidelines for performance of the routine mid-trimester fetal ultrasound scan. Ultrasound Obstet Gynecol. 2011; 37: 116-126.

11 E. Merz, K.-H. Eichhorn, C. von Kaisenberg, T. Schramm. Aktualisierte Qualitätsanforderungen an die weiterführende differenzierte Ultraschalluntersuchung in der pränatalen Diagnostik (= DEGUM-Stufe II) im Zeitraum von $18+0$ bis $21+6$ Schwangerschaftswochen. [Updated Quality Requirements Regarding Secondary Differentiated Ultrasound Examination in Prenatal Diagnostics ( = DEGUM Level II) in the Period from $18+0$ to $21+6$ Weeks of Gestation]. Ultraschall in Med 2012; DOI http://dx.doi.org/10.1055/ s-0032-1325500

Softmarker (insbesondere \#12 und \#16)

12 Benacerraf BR. The history of the secondtrimester sonographic markers for detecting fetal Down syndrome, and their current role in obstetric practice. Prenat Diagn. 2010;30: 644-652. Review.

13 http://www.fetalmedicine.com/fmf/FMF-german.pdf (Seite 72, o Tabelle2). Die Tabelle stützt sich auf die zwei Arbeiten \# 12 und 13 , zusammengefasst in \#14:

14 Nyberg DA, Souter VL, El-Bastawissi A, Young $\mathrm{S}$, Luthhardt $\mathrm{F}$, Luthy DA. Isolated sonographic markers for detection of fetal Down syndrome in the second trimester of pregnancy. J Ultrasound Med. 2001; 20: 1053-1063.

15 Bromley B, Lieberman E, Shipp TD, Benacerraf BR. The genetic sonogram: a method of risk assessment for Down syndrome in the second trimester. J Ultrasound Med. 2002; 21: 1087-1096; quiz 1097-1098.

16 Nicolaides KH. Screening for chromosomal defects. US Obstet Gynecol 2003; 21: 313-321. 